Research Article

\title{
LncRNA NKILA Promotes Cardiomyocytes Apoptosis by Targeting miR22-3p-TXNIP Signal Axis to Inhibit Proliferation, Migration, and Invasion of Cardiomyocytes under High Glucose-Induced Condition
}

\author{
Yan Zhao $\mathbb{D}^{1},{ }^{1}$ Wenjuan Ming $\mathbb{D}^{1},{ }^{1}$ Xiaohuan $M a \mathbb{D}^{1},{ }^{1}$ Yuchan Zheng $\mathbb{D}^{1},{ }^{1}$ and Yanli Yan $\mathbb{D}^{2}$ \\ ${ }^{1}$ School of Sports and Health, Nanjing Sport Institute, Nanjing, Jiangsu 210014, China \\ ${ }^{2}$ Department of Emergency Medicine and Critical Care, Shanghai East Hospital, Tongji University School of Medicine, \\ Shanghai 200120, China \\ Correspondence should be addressed to Yanli Yan; yanliyan77@hotmail.com
}

Received 30 November 2020; Revised 27 January 2021; Accepted 11 March 2021; Published 29 March 2021

Academic Editor: Qinhu Zhang

Copyright (c) 2021 Yan Zhao et al. This is an open access article distributed under the Creative Commons Attribution License, which permits unrestricted use, distribution, and reproduction in any medium, provided the original work is properly cited.

This study aims to investigate the molecular mechanism of LncRNA NKILA underlying promoting cardiomyocytes apoptosis and relevant diabetic cardiomyopathy. We utilized high concentration of glucose to induce human cardiomyocytes cell line AC16 to imitate diabetic cardiomyopathy. And then, we performed high-throughput big data analysis, RT-PCR, and western blot assays to evaluate the expression levels of associated mRNA and protein. Cell apoptosis was tested by Annexin V-FITC. The proliferation, migration, and invasion of AC16 cells were examined by CCK8 assay, colony formation assay, EdU assay, wound healing test, and transwell chamber assay. We utilized statistical analysis and luciferase activity assay to analyze the interaction of relevant genes. LncRNA NKILA was highly expressed in AC16 cells induced by high glucose and inhibited AC16 cell proliferation, migration, and invasion by inducing cell apoptosis. Luciferase activity assay demonstrated that LncRNA NKILA binds to miR22-3p. The influence of LncRNA NKILA on AC16 cell proliferation, migration, and invasion could be reversed by miR22-3p. Luciferase activity assay demonstrated that TXNIP was a target of miR-22-3p in AC16 cells, and all the effects of TXNIP on AC16 cell proliferation, migration, and invasion could be abolished by miR22-3p. These results provided comprehensive data about a novel molecular mechanism of LncRNA NKILA promoting cardiomyocytes apoptosis: LncRNA NKILA performed its function in AC16 cells under high glucose-induced condition by targeting mir-22-3p-TXNIP signal axis, which indicated that LncRNA NKILA may play a crucial role in diabetic cardiomyopathy.

\section{Introduction}

The development and progress of diabetes will lead to a series of complications, affecting most of the main organs of the human body [1]. Big data statistical analysis shows there is growing evidence that diabetes complications are one of the leading causes of death in many countries [2, 3]. As a complication of diabetes mellitus, diabetic cardiomyopathy (DCM) is an independent risk factor related to heart failure, even after the treatment of coronary heart disease (CAD), hypertension, or vascular heart disease [4]. Increasing research and statistical analysis suggest that the development of DCM is a process involving multiple metabolic disorders, including myocardial insulin resistance and inhibition of glucose oxidation, resulting in increasing lipid metabolism, hyperlipidemia, myocardial insulin resistance, and hyperinsulinemia [5]. Clinical research shows that DCM is an important risk factor and one of the main reasons for significant heart failure in about $12 \%$ of diabetic patients worldwide $[6,7]$. Reversing DCM can prevent heart stroke and reduce the incidence rate of cardiovascular disease (CVD). Due to the lack of understanding of the basic mechanisms of DCM pathogenesis, it resulted in the absence of an effective clinical prognosis and treatment strategy. 
Currently, a large number of efforts have shown that noncoding RNA (ncRNAs) is a key factor of diabetic vascular complications [8]. As a subgroup of ncRNAs, long noncoding RNAs (lncRNAs) are larger than 200 nucleotides in length [9]. Recently, with more and more lncRNAs proving to be key participants in DCM, their regulation may contribute to the control of such diseases $[10,11]$. Nuclear factor $-\kappa \mathrm{B} \quad(\mathrm{NF}-\kappa \mathrm{B})$ interacting long noncoding RNA (LncRNA NKILA) is a well-studied tumor suppressor lncRNA in a variety of malignancies [12, 13]. NF- $\kappa \mathrm{B}$ interacting lncRNA (NKILA) was first identified in breast cancer, located in the cytoplasm [14]. In cytoplasm, NKILA binds to the NF- $\kappa \mathrm{B}$ complex to form a stable ternary complex that blocks the $\mathrm{I} \kappa \mathrm{B}$ phosphorylation site, leading to inhibition of $\mathrm{I} \kappa \mathrm{B}$ phosphorylation and NF- $\kappa \mathrm{B}$ activation.

MicroRNAs (miRNAs) are a class of endogenous small molecule noncoding RNAs that play an important regulatory role in many important biological processes [15]. At present, the role of miRNAs in diabetes mellitus and its cardiovascular complications has not been fully studied [16-18]. However, with the increasing evidence showing the important role of miRNAs, its importance in diabetes and its cardiovascular complications are now receiving more and more attention. For instance, circulating miRNAs are currently considered as potential biomarkers for a variety of diseases, including diabetes [19]. Thioredoxin interaction protein gene (TXNIP) is involved in the regulation of oxidative stress-sensitive signaling pathways and is associated with insulin resistance and impaired islet cell function $[20,21]$. Because of its role in insulin resistance and islet cell function, it is thought to be involved in DCM pathogenesis.

In this study, we utilized high concentration of glucose to induce human cardiomyocytes cell line AC16 to imitate diabetic cardiomyopathy. And we investigated the role of LncRNA NKILA in promoting cardiomyocytes apoptosis in vitro and found out that LncRNA NKILA performed its function in promoting AC16 cell apoptosis by targeting mir22-3p-TXNIP signal axis, which suggested a new therapeutic strategy against diabetic cardiomyopathy.

\section{Materials and Methods}

2.1. Reagent. DMEM medium (SH30022.01 B), FBS (SH30 087.01), penicillin (SH30010), and PBS (SH30256.01 B) were brought from $\mathrm{GE}^{\mathrm{TM}}$ Hyclone company. Lipofectamine $\mathrm{T}^{\mathrm{TM}}$ RNAiMAX transfection reagent was brought from Invitrogen (13778-075, USA). Antibodies: anti- $\beta$-actin (ab8227), anti-Bax (ab32503), anti-Bcl-2 (ab32124), anti-cleaved caspase-3 (ab2302), anti-cleaved caspase-9 (ab2324), antiCOX-2 (ab15191), anti-MMP-2 (ab97779), anti-MMP-9 (ab38898), and anti-TXNIP (ab188865) were purchased from Abcam Co., Ltd, Cambridge, UK.

2.2. Cell Culture and Vector Transfection. AC16 cells were maintained in the DMEM medium (SH30022.01 B, Hyclone, USA) containing with 10\% FBS (SH30087.01, Hyclone, USA), $100 \mathrm{U} / \mathrm{ml}$ penicillin (SH30010, Hyclone, USA), and $100 \mathrm{mg} / \mathrm{ml}$ streptomycin in a humidified atmosphere at $37^{\circ} \mathrm{C}$ with $5 \% \mathrm{CO}_{2} .24$ hours before transfection, AC16 cells were transferred into the Opti-MEM culture medium. NKILA shRNA or miR22-3p overexpression vector or both were transfected into AC16 cells to silence expression using Lipofectamine 2000 (Cat. No. 11668019, Invitrogen, USA) according to the manufacturer's instruction. In brief, $1.25 \mu \mathrm{l}$ NKILA shRNA $(20 \mu \mathrm{M})$ or $1 \mu \mathrm{g}$ of miR22-3p vector was diluted with $100 \mu \mathrm{l}$ Opti-MEM, called as fluid A. Fluid B was Opti-MEM containing $1 \mu \mathrm{l}$ of Lipofectamine 2000 and dissolved for 5 minutes before being mixed with fluid A. Transfection reagent was added into AC16 cells and was plated in 24-well plates (Cat. No. 3548, Corning, USA), which was transferred into Opti-MEM medium 24 hours before transfection. After 4 to 6 hours of reaction, the culture medium was changed into DMEM full-culture medium. Gene expression test was performed by qPCR 36 hours after transfection. Test of cell function, proliferation, cell cycle, and migration were performed in 48 hours.

2.3. Real-Time PCR. Real-time PCR was performed to verify the efficiency of relevant shRNA or overexpression vector in AC16 cells. Total mRNA was isolated from AC16 cells using the Trizol reagent (\#15596018) (Life Technologies, USA) and then reversed transcribed by the QuantiTect Reverse Transcription Kit (\#205313) (Qiagen, Shanghai, China). Real-Time PCR was performed by the StepOnePlus system (Applied Biosystem, USA) using Thermo Fisher Scientific Maxima SYBR Green/ROX qPCR Master Mix assay $(2 \times)$ (\#K0221). Primer sequences are demonstrated in Table 1.

2.4. Western Blot. To detect the cellular level of target proteins, protein extracted from AC16 cells was detected by western blot. Whole cell lysates were extracted by using the lysis buffer: $50 \mathrm{mM}$ Tris $\mathrm{pH}$ 7.4, $150 \mathrm{mM} \mathrm{NaCl}, 1 \mathrm{mM}$ EDTA, $1 \%$ Triton, and $10 \%$ glycerol along with protease and phosphatase inhibitor cocktail (Roche, Basel, Switzerland). Protein concentrations were determined by the Bradford assay. Soluble proteins $(30-40 \mu \mathrm{g})$ were subjected to SDSpolyacrylamide gel electrophoresis. Separated proteins were electrophoretically transferred onto polyvinylidene difluoride (PVDF) membranes (Millipore, Billerica, MA, USA). Primary antibodies utilized in the present study were diluted into $5 \%$ nonfat milk as $1: 500$.

2.5. Cell Proliferation Test. Cell proliferation test was performed according to CCK8 manuscript in day 0 , day 1 , day 2 , and day 4 after transfection.

2.6. Colony Formation Assay. Trypsin was digested into individual cells and suspended in medium. Each group was inoculated with $10 \mathrm{ml}$ culture medium with 200 cells per dish, and the cells were evenly dispersed by gentle shaking. Routine culture was performed for 3 weeks. When visible clones appeared in the Petri dish, the medium was discarded, fixed with $4 \%$ paraformaldehyde for $15 \mathrm{~min}$, dyed with $0.1 \%$ crystal violet dye for $10 \mathrm{~min}$, and washed with PBS; the number of clones was counted. 
Table 1: Primer sequences.

\begin{tabular}{lc}
\hline Gene & Sequence \\
\hline U6 & F $5^{\prime}$-CTCGCTTCGGCAGCACAT-3 ${ }^{\prime}$ \\
R $5^{\prime}$-TTTGCGTGTCATCCTTGCG-3' \\
\hline \multirow{2}{*}{ NKILA } & F $5^{\prime}$-AACCAAACCTACCCACAACG-3' \\
& R $5^{\prime}$-ACCACTAAGTCAATCCCAGGTG-3' \\
\hline \multirow{2}{*}{ miR22-3p } & F $5^{\prime}$-GTTCTTCAGTGGCAAGC-3' \\
& R $5^{\prime}$-GAACATGTCTGCGTATCTC-3' \\
\hline \multirow{2}{*}{ TXNIP } & F $5^{\prime}$-CAGCAGTGCAAACAGACTTCGG-3' \\
& R $5^{\prime}$-CTGAGGAAGCTCAAAGCCGAAC-3' \\
\hline \multirow{2}{*}{ GAPAH } & F $5^{\prime}$-GTGGACCTGACCTGCCGTCT-3' \\
& R $5^{\prime}$-GGAGGAGTGGGTGTCGCTGT-3' \\
\hline
\end{tabular}

2.7. EdU Staining Assay. $5 \times 10^{4}$ cells were inoculated on 96well plates. After $48 \mathrm{~h}$, the cells were digested and collected and then incubated in the medium containing EdU for $2 \mathrm{~h}$. After PBS washing, $4 \%$ paraformaldehyde was fixed for $30 \mathrm{~min}$. Then, reagents $B, C, D$, and $E$ were added, respectively, according to the instructions of kit (Guangzhou Ruibo Biotechnology Co., Ltd.) for incubation, followed by PBS washing. And finally, it was incubated with hoechst 33342 staining solution at room temperature for $30 \mathrm{~min}$, washed with PBS, observed and photographed under fluorescence microscope, and analyzed with Image $J$.

2.8. Cell Apoptosis by PI-Annexin V Assay. Proportion of cell apoptosis after transfection was tested by PI-Annexin V assay and displayed by flow cytometry. In brief, the cells in the logarithmic growth stage were seeded in a 6-well plate with a density of $2 \times 10^{5} /$ well and cultured for $48 \mathrm{~h}$. After washing with precooled PBS twice, the supernatant was discarded after $4000 \mathrm{~g}$ of centrifuged cell suspension for $3 \mathrm{~min}$, and $400 \mu \mathrm{l}$ Annexin v-FITC staining solution was added and incubated on ice under dark for $15 \mathrm{~min}$. Then, $10 \mu \mathrm{l}$ PI dye solution was added and mixed and incubated under dark for $5 \mathrm{~min}$. The apoptosis was detected by flow cytometry, and the excitation light wavelength was $488 \mathrm{~nm}$. The experiment was repeated for 3 times, and the average value of the three experiments data was taken.

2.9. Wound Healing Assay. Wound healing assay was performed as previously described [22].

2.10. Transwell Chamber Assay. Cells in the logarithmic growth stage were starved for 24 hours and digested the next day, centrifugated, and resuspended, with a final concentration of $2 \times 10^{5} / \mathrm{ml} .0 .2 \mathrm{ml}$ of suspension was added to the transwell upper chamber, and $700 \mathrm{ml}$ of precooled DMEM cell culture medium containing $10 \%$ FBS was added to the lower chamber. The cells were cultured in a cell incubator containing $5 \% \mathrm{CO}_{2}$ at a temperature of $37^{\circ} \mathrm{C}$. After 24 hours, the transwell chamber was removed and the cells in the upper chamber and the basement membrane were swabbed with wet cotton swabs. The cells were fixed with methanol for $30 \mathrm{~min}$ and stained with $0.1 \%$ crystal violet dye for $20 \mathrm{~min}$. Five fields $(100 \times)$ were randomly selected to count the number of transmembrane cells.
2.11. Nuclear and Cytoplasmic Separation Assay. The cells were resuspended with hypotonic buffer containing protease inhibitor and RNase inhibitor (n8080119, Thermo Fisher Scientific) (10 mm HEPES (pH 7.5), $0.5 \mathrm{~mm}$ DTT, $10 \mathrm{mM}$ $\mathrm{KCl}$, and $1.5 \mathrm{mM} \mathrm{MgCl}_{2}$ ). After incubation on ice for 10 minutes, centrifuge at $1000 \mathrm{~g}$ at $4^{\circ}$ for 10 minutes. The supernatant was further centrifuged at $15000 \mathrm{~g}$ for 15 minutes to obtain the cytoplasm. The precipitate was rinsed twice with hypotonic buffer B (10 mm HEPES ( $\mathrm{pH}$ 7.5), $10 \mathrm{mM} \mathrm{KCl}, 1.5 \mathrm{mM} \mathrm{MgCl} 2,0.5 \mathrm{mM}$ DTT, and $0.5 \%$ Nonidet P-40), incubated at $4^{\circ} \mathrm{C}$ for $30 \mathrm{~min}$, rotated gently, centrifuged at $6000 \mathrm{~g}$ at $4^{\circ} \mathrm{C}$ for $10 \mathrm{~min}$, and rinsed once with hypotonic buffer, Ripa buffer containing protease inhibitor, and RNase inhibitor (50 mM Tris $\mathrm{HCl}$ (pH 7.5), $1500 \mathrm{mM}$ $\mathrm{KCl}, 1 \%$ Nonidet $\mathrm{P}-40,0.5 \%$ sodium hydroxide, $0.1 \%$ SDS, and $1 \mathrm{mM}$ EDTA, pH 8.0) heavy suspension. Incubate at $4^{\circ} \mathrm{C}$ for $30 \mathrm{~min}$, rotate gently at the same time, centrifugate at $15000 \mathrm{~g}$ for $20 \mathrm{~min}$, and the supernatant is the nuclear part.

2.12. Luciferase Reporter Gene Technique. Dual Luciferase Gene Reporter Assays was previously described [23].

2.13. Statistical Analysis. All experiments in this study were repeated at least two times, and average values of three experiments were presented as the mean standard deviation (SD) calculated by STDEV formula in Excel. The significance of all data was estimated by Tukey's multiple comparison test in the ANOVA analysis using the SigmaStat 3.5 software. Importantly, statistical significance was accepted when $P<0.05$.

\section{Results}

3.1. LncRNA NKILA Was Highly Expressed in AC16 Cells Induced by High Glucose. We performed RT-PCR assay to analyze the expression level of NKILA mRNA in AC16 cells under high glucose-induced condition. It was found that the expression level of NKILA mRNA increased significantly with the increase in the time of hyperglycemia induction (Figure 1(a)). Furthermore, it was found that the increase in NKILA mRNA expression level was dose-dependent on the glucose concentration (Figure 1(b)). And without surprising, sh-NKILA was able to knockdown NKILA mRNA expression level under high glucose-induced condition (Figure 1(c)).

3.2. Knocking Down LncRNA NKILA Reversed the Apoptosis of AC16 Cells Induced by High Glucose and Promoted Its Proliferation. It was found that knocking down LncRNA NKILA reversed the inhibition of AC16 cells vitality caused by high glucose when we performed CCK8 assay (Figure 2(a)). This reversion was observed in AC16 cells colony forming ability and AC16 cells proliferation ability as well (Figures 2(b) and 2(c)). To evaluate cell apoptosis, we performed Annexin V-FITC assay and found that high glucose induced AC16 cells apoptosis which could be abolished by sh-NKLA (Figure 2(d)). Furthermore, apoptosis protein makers: BAX, cleaved caspase- 3 , and cleaved caspase- 9 were induced by high glucose, while anti- 


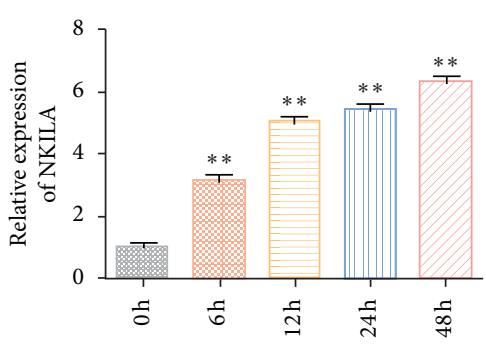

(a)

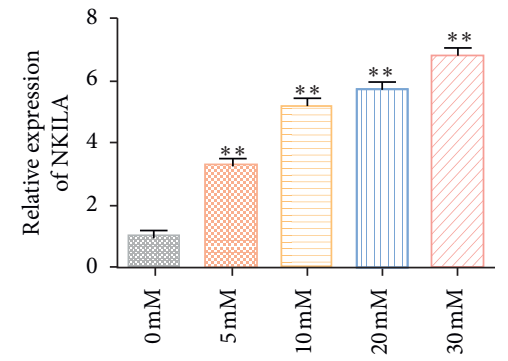

(b)

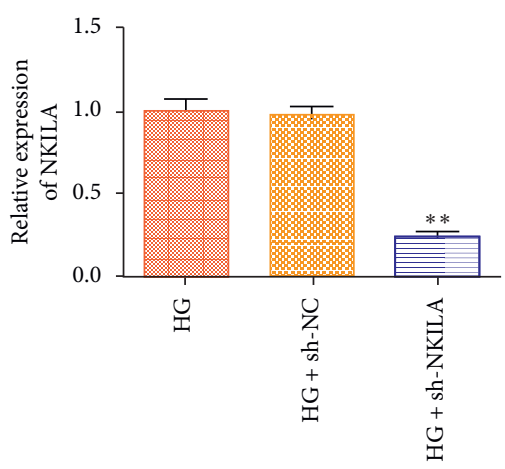

(c)

FIGURE 1: LncRNA NKILA was highly expressed in AC16 cells induced by high glucose. (a) the expression level of LncRNA NKILA mRNA detected by RT-PCR after inducing on $30 \mathrm{mM}$ glucose at the indicated time in AC16 cells; (b) the expression level of LncRNA NKILA mRNA detected by RT-PCR after inducing with various concentrations of glucose $(0,5,10,20$, and $30 \mathrm{mM}$ ) for $48 \mathrm{~h}$ in AC16 cells; (c) the expression level of LncRNA NKILA mRNA detected by RT-PCR after transfecting indicated plasmid in AC16 cells under high glucose-induced condition. Data are representative of three independent experiments and were analyzed by unpaired $t$-test. Error bars denote $\mathrm{SD} .{ }^{*} P<0.05$; ${ }^{* *} P<0.01 ;{ }^{* * *} P<0.001$.

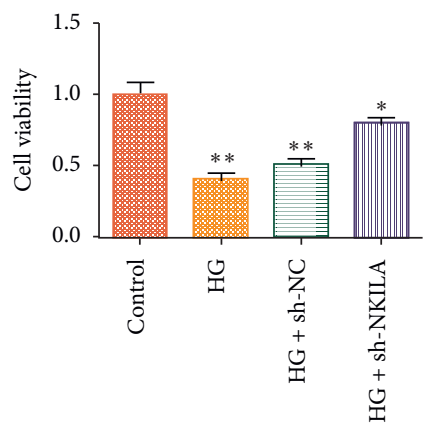

(a)
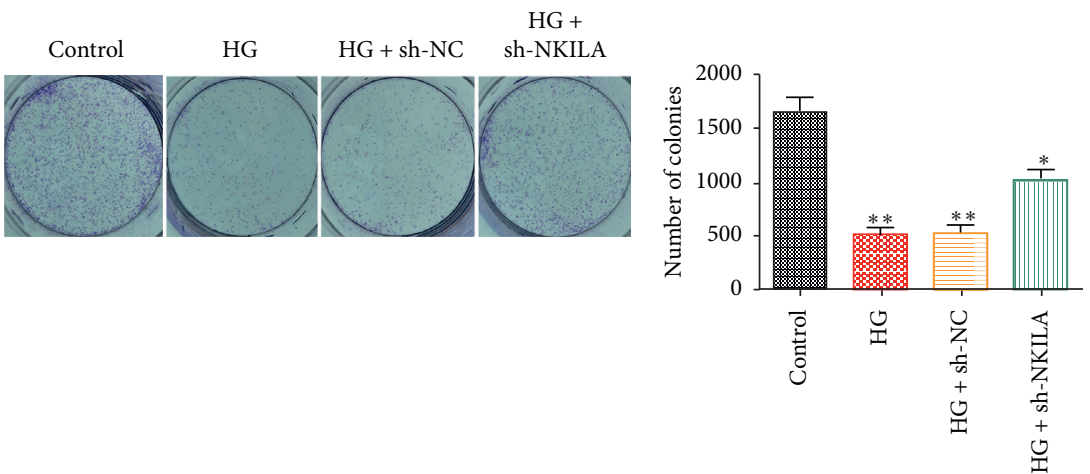

(b)
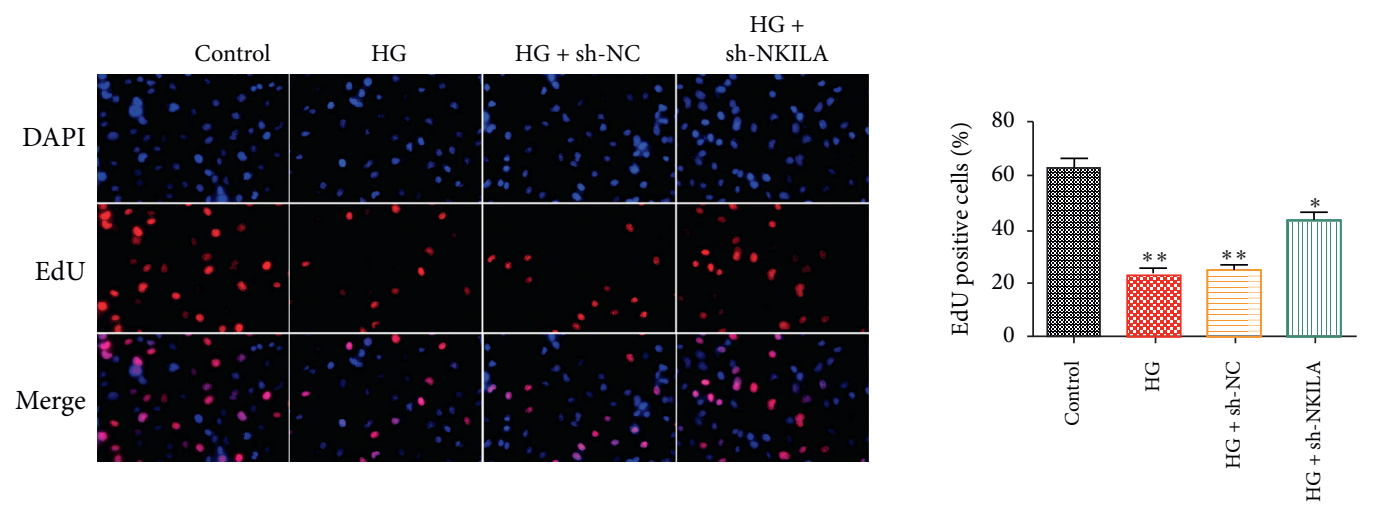

(c)

Figure 2: Continued. 


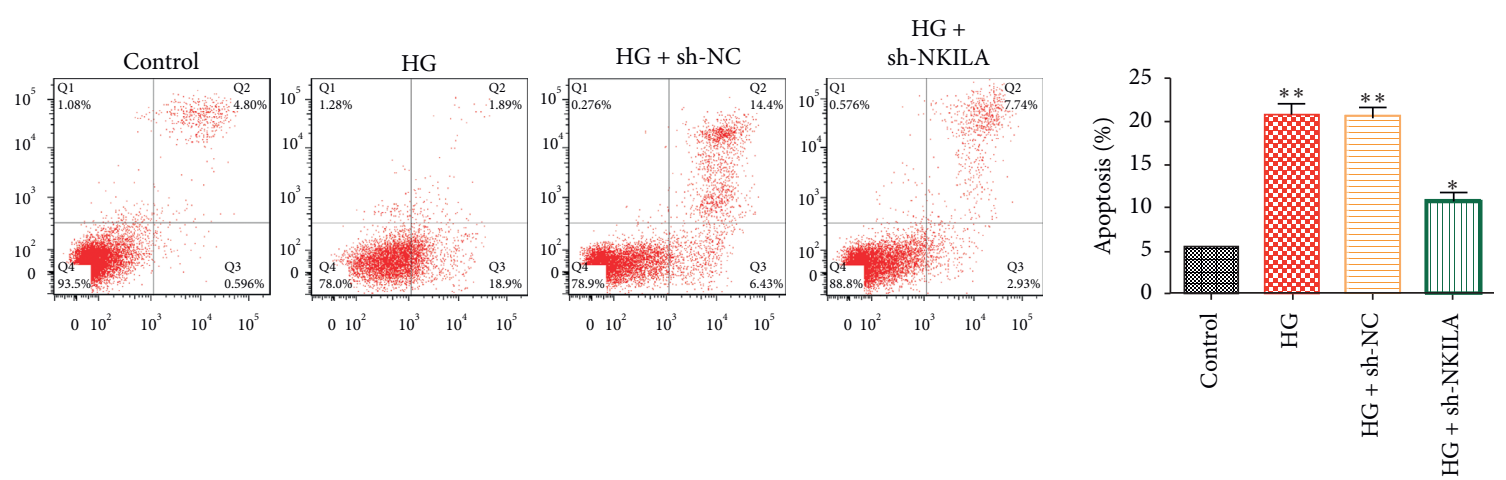

(d)

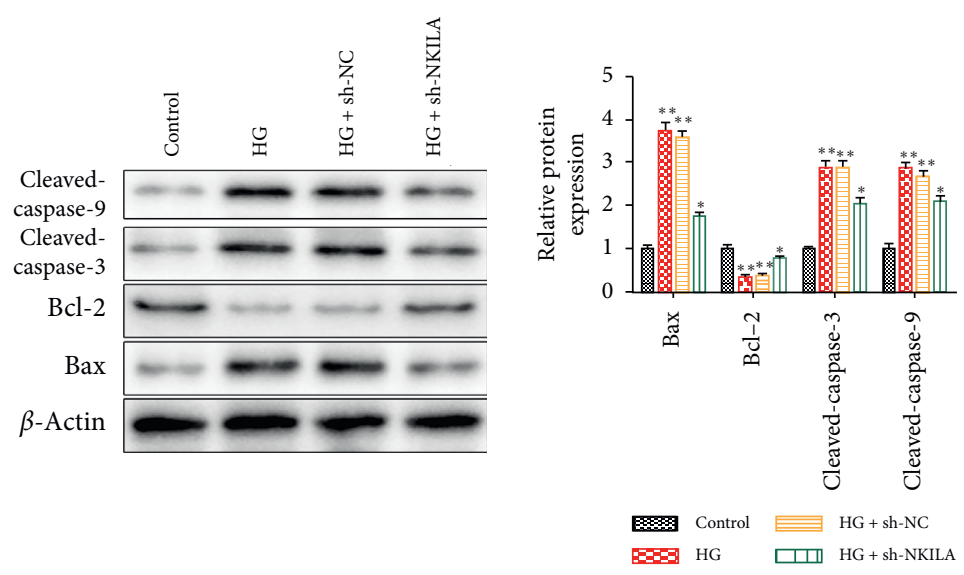

(e)

FIGURE 2: Knocking down LncRNA NKILA reversed the apoptosis of AC16 cells induced by high glucose and promoted its proliferation. (a) AC16 cells viability detection by CCK8 assay of knocking down LncRNA NKILA under high glucose-induced condition and indicated plasmids were transfected in AC16 cells; (b, c) AC16 cells colony forming ability and proliferation ability detection by colony forming assay and EDU assay of knocking down LncRNA NKILA under high glucose-induced condition and indicated plasmids were transfected in AC16 cells; (d) AC16 cells apoptosis detection by Annexin V-FITC assay of knocking down LncRNA NKILA under high glucose-induced condition and indicated plasmids were transfected in AC16 cells; (e) cell apoptosis protein makers expression level detection by western blot assay after transfecting indicated plasmids in AC16 cells and indicated antibodies were added during western blot assay. Data are representative of three independent experiments and were analyzed by the unpaired $t$-test. Error bars $\operatorname{denote} \mathrm{SD} .{ }^{*} P<0.05$; ${ }^{* *} P<0.01$; ${ }^{* * *} P<0.001$.

apoptosis protein $\mathrm{Bcl} 2$ was inhibited (Figure 2(e)). And all these effects on protein could be reversed by knocking down LncRNA NKILA. Taken together, these data indicated high glucose-induced AC16 cells apoptosis and inhibited AC16 cells proliferation through increasing NKLA expression.

\subsection{Knocking Down LncRNA NKILA Promoted AC16 Cells} Migration and Invasion under High Glucose-Induced Condition. To evaluate influence of knocking down LncRNA NKILA of AC16 cells migration and invasion under high glucose-induced condition, we performed wound healing test and transwell chamber assay. As shown in Figures 3(a) and 3(b), high glucose-induced (HG) significant inhibited cell migration and invasion compared with control group, and this inhibition was reversed by knocking down LncRNA NKILA (HG+sh-NKILA). Furthermore, migration and invasion protein makers: Cox-2, MMP-2, and MMP-9 were significantly inhibited by HG compared with control group, while all these effects on protein could be reversed by knocking down LncRNA NKILA (Figure 3(c)). Taken together, these data indicated high glucose inhibited AC16 cells migration and invasion through increasing NKLA expression.

3.4. LncRNA NKILA Performed Its Function in AC16 Cells under High Glucose-Induced Condition by Binding miR22-3p. To figure out the molecular mechanism of LncRNA NKILA in regulating AC16 cells proliferation, migration, and invasion, we searched starBase and found out the match region between LncRNA NKILA and miR22-3p (Figure 4(b)). And this result was mutually supportive of the high expression of LncRNA NKILA in the cytoplasm (Figure 4(a)). Furthermore, we utilized luciferase activity assay to analyze the interaction between LncRNA NKILA and miR22-3p. Compared with control group (NC mimic), miR22-3p overexpressing (miR22-3p mimic) significantly inhibited NKILA luciferase activity, which was abolished by NKILA mutation (Figure 4(b)), suggesting LncRNA NKILA binds to 

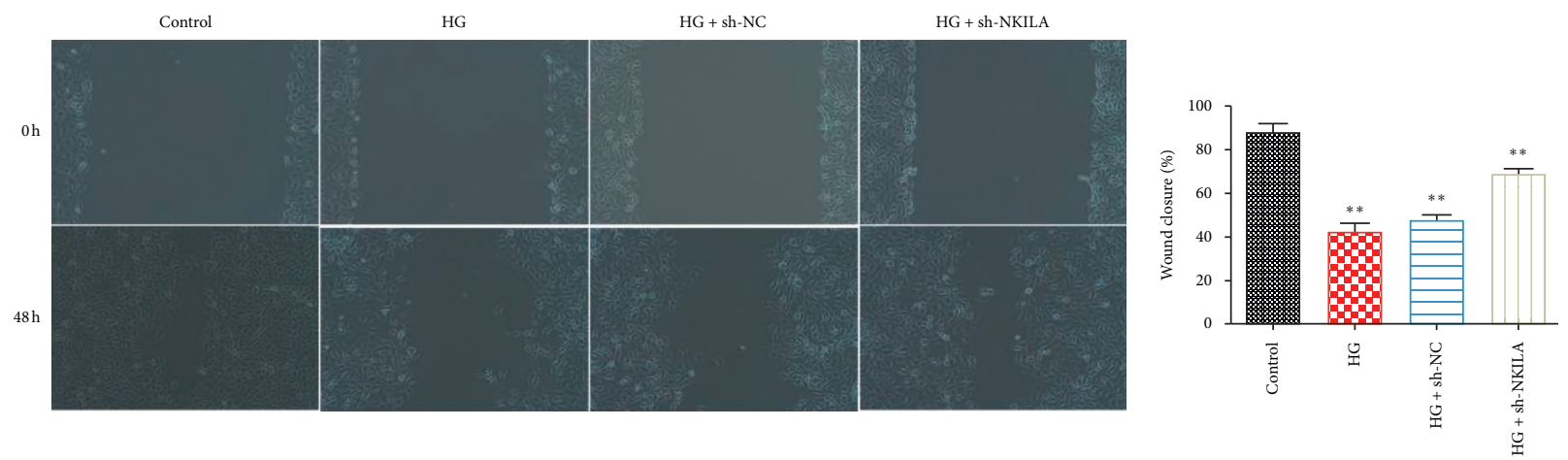

(a)
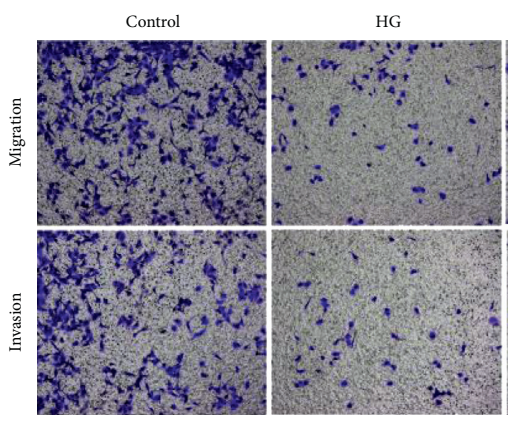

$\mathrm{HG}+\mathrm{sh}-\mathrm{NC}$

HG + sh-NKILA
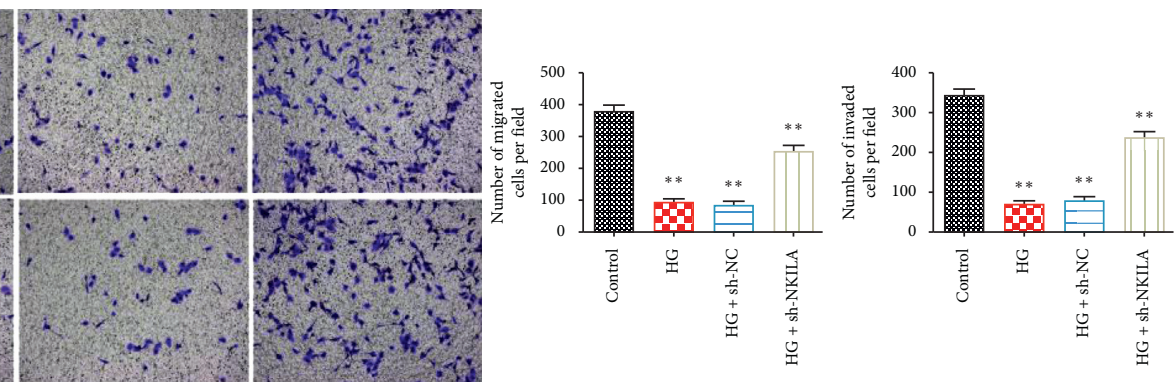

(b)

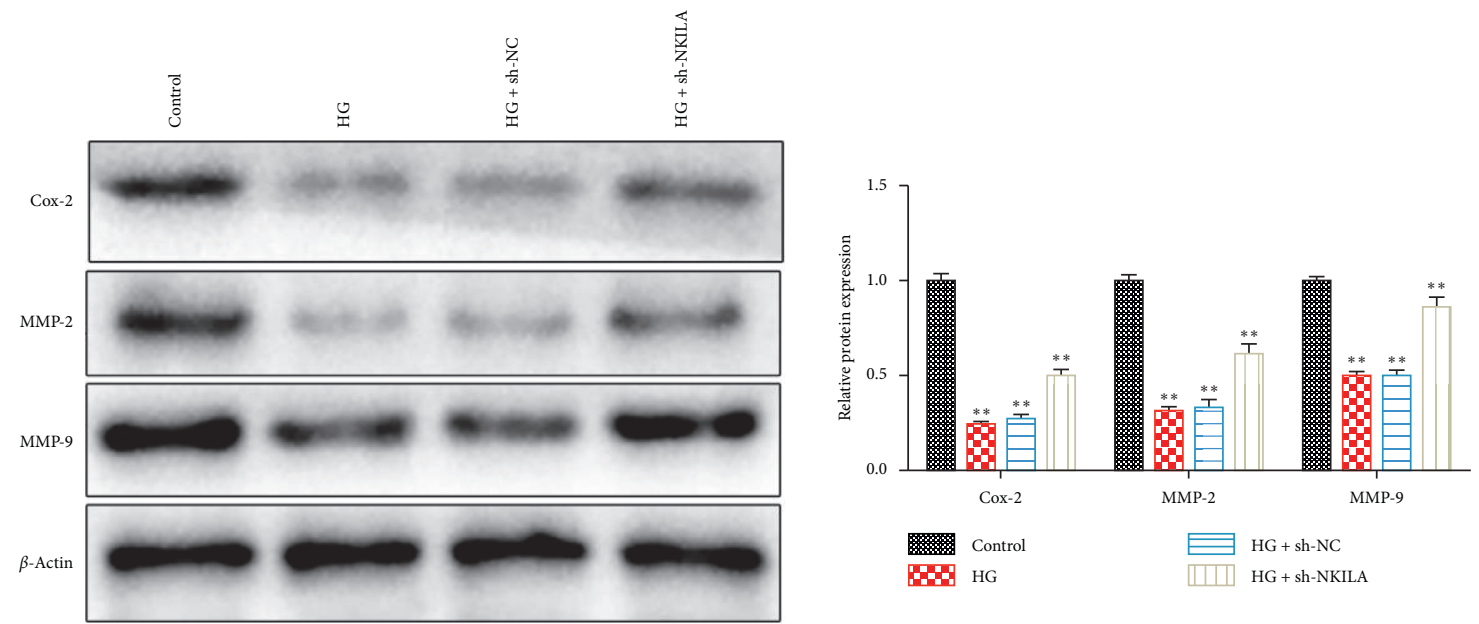

(c)

FIGURE 3: Knocking down LncRNA NKILA promoted AC16 cells migration and invasion under high glucose-induced condition. (a, b) AC16 cells migration and invasion detection by wound healing test and transwall chamber assay of knocking down LncRNA NKILA under high glucose-induced condition and indicated plasmids were transfected in AC16 cells; (c) cell migration and invasion protein makers expression level detection by western blot assay after transfecting indicated plasmids in AC16 cells and indicated antibodies were added during western blot assay. Data are representative of three independent experiments and were analyzed by the unpaired $t$-test. Error bars denote SD. ${ }^{*} P<0.05 ;{ }^{* *} P<0.01 ;{ }^{* * *} P<0.001$.

miR22-3p (Figure 4(c)). The expression level of miR22-3p mRNA decreased significantly with the increase in time of high glucose induction (Figure $4(\mathrm{~d})$ ), and the decrease in miR22-3p mRNA expression level was dose-dependent on the glucose concentration (Figure 4(e)). Consistent with this, knocking down LncRNA NKILA significant increased miR22-3p mRNA expression level (Figure 4(f)).

These data indicated that LncRNA NKILA performed its function in AC16 cells under high glucose-induced condition by binding miR22-3p. To prove this hypothesis, we evaluated the influence of miR22-3p on AC16 cells proliferation, migration, and invasion under high glucose-induced condition. First, we overexpressed miR22-3p in AC16 cells (Figure 5(a)). Then, we performed CCK8 assay and found that, under high glucose-induced condition, miR22-3p overexpressing ( $\mathrm{HG}+$ miR22-3p mimic) significantly increased AC16 cells viability compared with control group (HG + NC mimic) (Figure 5(b)). And this degree of increase cautilized by miR22-3p was 


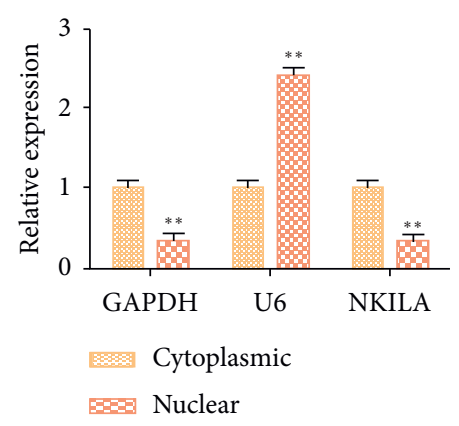

(a)

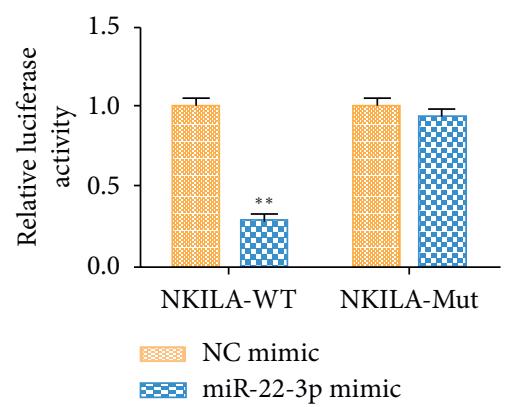

(c)

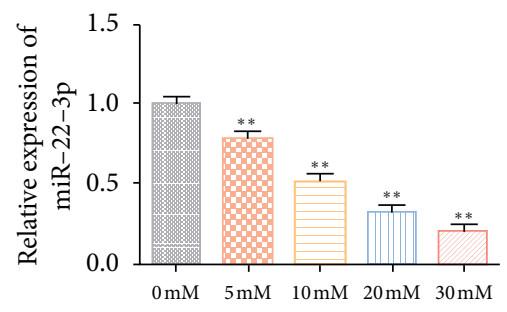

(e)

$\begin{array}{lr}\text { NKILA WT } & 5^{\prime} \text {-cccaUUUUACAGAGCGGGCAGCUg-3' } \\ \text { Hsa-miR-22-3p } & 3^{\prime} \text {-ugucAAGAAG---UUGGCCGUCGAa-5' } \\ \text { NKILA Mut } & 5^{\prime} \text {-cccaUUUUACAGAGCGGGCAGCUg-3' }\end{array}$

(b)

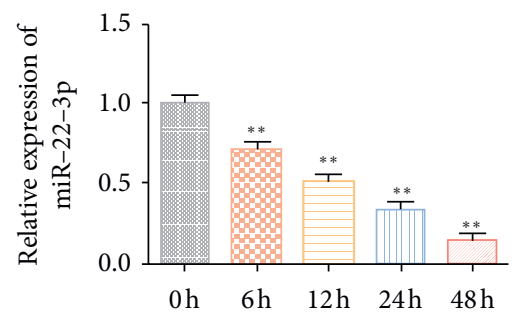

(d)

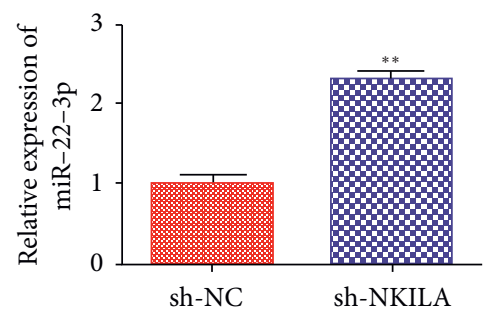

(f)

FIGURE 4: LncRNA NKILA in regulating AC16 cells proliferation, migration, and invasion. (a) Under high glucose-induced condition, the expression level of LncRNA NKILA mRNA in AC16 cells. Nucleus and cytoplasm was detected by RT-PCR after nuclear and cytoplasmic separation assay; (b) aligned region between sequence of LncRNA NKILA and miR22-3p and sequence of LncRNA NKILA mutation (NKILA Mut); (c) luciferase activity assay to analyze the interaction between LncRNA NKILA (wt: wild type or Mut: mutation) and miR22$3 p$ under high glucose-induced condition; (d) the expression level of miR22-3p mRNA detected by RT-PCR after inducing on 30 mM glucose at the indicated time in AC16 cells; (e) the expression level of miR22-3p mRNA detected by RT-PCR after inducing with various concentrations of glucose $(0,5,10,20$, and $30 \mathrm{mM})$ for $48 \mathrm{~h}$ in AC16 cells; (f) the expression level of miR22-3p mRNA detected by RT-PCR after transfecting indicated plasmid in AC16 cells under high glucose-induced condition. Data are representative of three independent experiments and were analyzed by the unpaired $t$-test. Error bars denote SD. ${ }^{*} P<0.05 ;{ }^{* *} P<0.01 ;{ }^{* * *} P<0.001$.

observed in AC16 cells colony forming ability and AC16 cells proliferation ability as well (Figures 5(c) and 5(d)). To evaluate cell apoptosis, we performed Annexin V-FITC assay and found that high glucose-induced AC16 cell apoptosis could be rescued by miR22-3p overexpressing (Figure 5(e)). Furthermore, under high glucose-induced condition, expression of apoptosis protein makers: BAX, cleaved caspase-3, and cleaved caspase- 9 were significant inhibited by miR22-3p overexpressing (HG + miR22-3p mimic) in AC16 cells compared with control group (HG + NC mimic), while expression of anti-apoptosis protein Bcl2 was induced (Figure 5(f)).

Next, we performed wound healing test chamber assay to analyze miR22-3p affect on migration and invasion of AC16 cells under high glucose-induced condition. As shown in Figures 6(a) and 6(b), high glucose-induced (HG) group significant inhibited cell migration and invasion compared with control group, and this inhibition was rescued by miR22-3p overexpressing ( $\mathrm{HG}+\mathrm{miR} 22-3 p$ mimic). In addition, migration and invasion protein makers: Cox-2, MMP-2, and MMP-9 were significantly inhibited by the HG group compared with control group, while all these effects on protein could be reversed by miR22-3p overexpressing $(\mathrm{HG}+\mathrm{miR} 22-3 p$ mimic) (Figure 6(c)). Taken together, these data indicated that, under high glucose-induced condition, LncRNA NKILA inhibited the proliferation, migration, and invasion of AC16 cells by binding miR22-3p. 

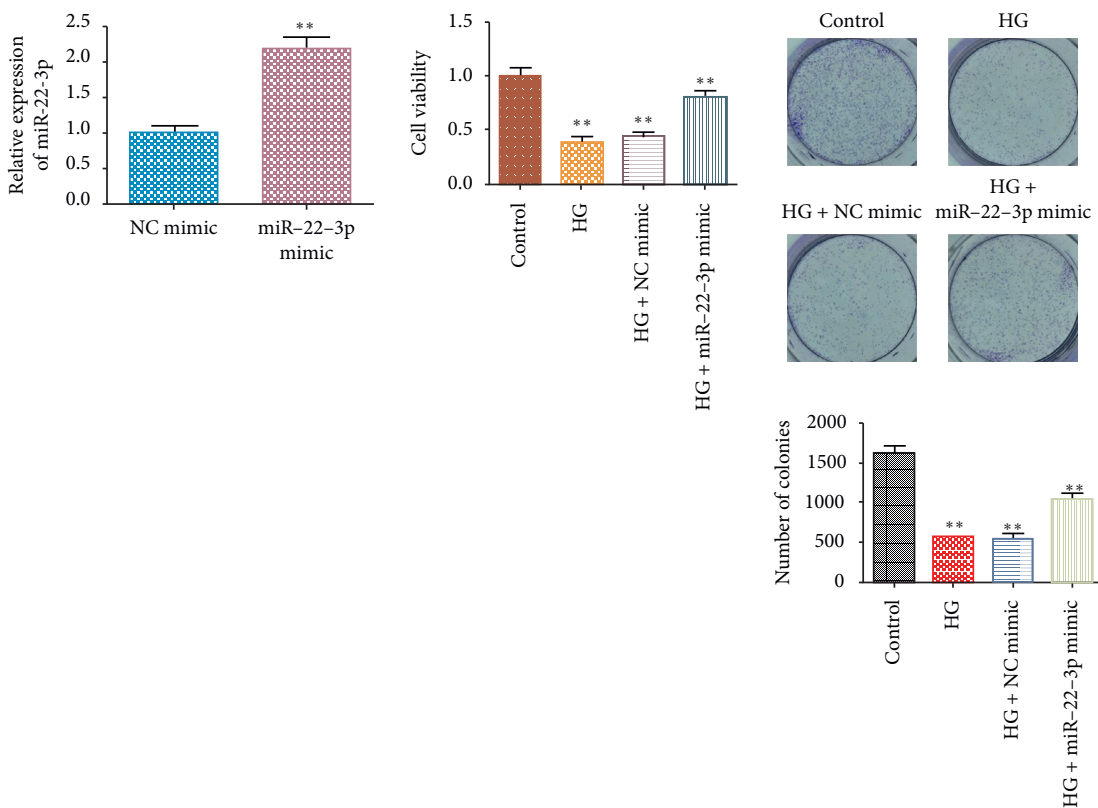

(a)

(b)

(c)
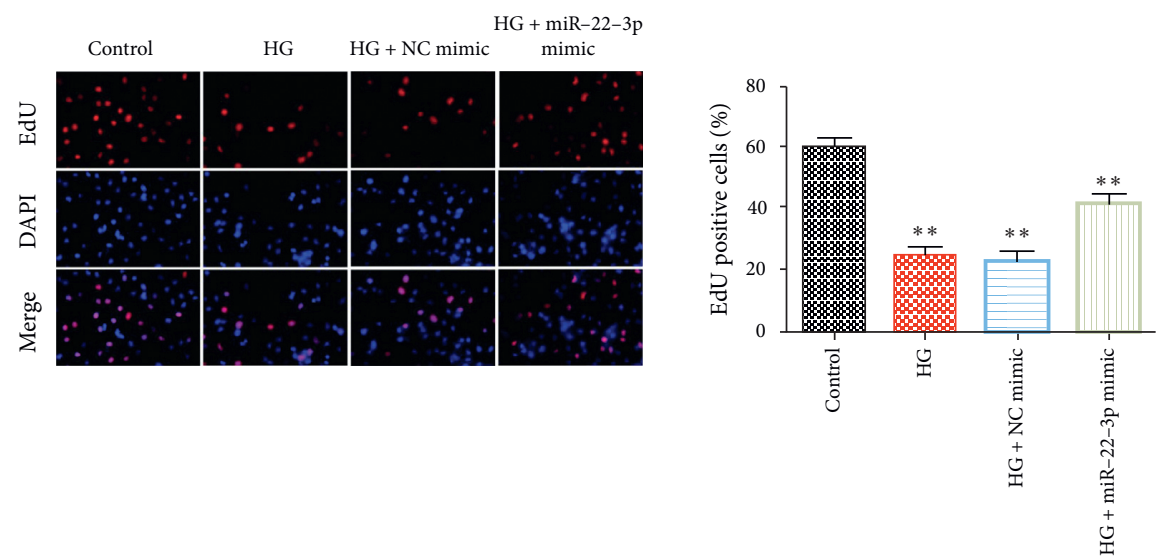

$\nLeftarrow \bullet$ Control

$\mathrm{E}=\mathrm{\Xi} \mathrm{BG}$

言 $\mathrm{HG}+\mathrm{NC}$ mimic

페ाता1) $\mathrm{HG}+\mathrm{miR}-22-3 \mathrm{p}$ mimic

(d)

Figure 5: Continued. 


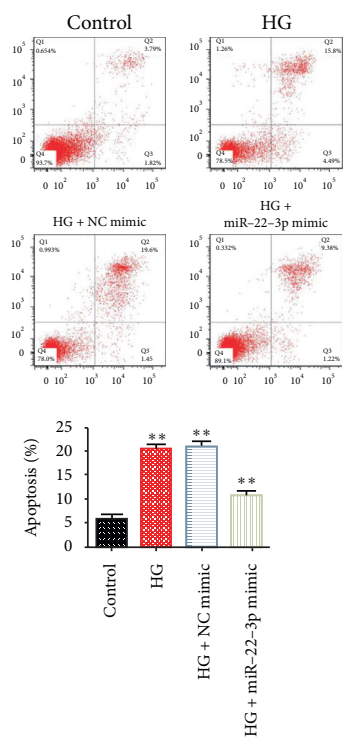

(e)
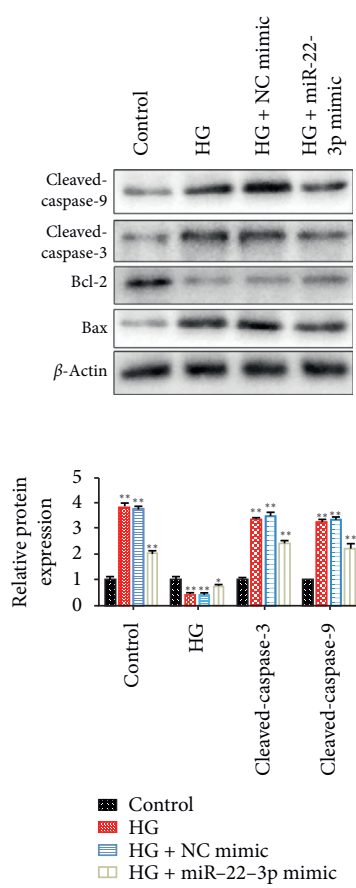

(f)

FIgURE 5: The influence of miR22-3p on AC16 cells proliferation, migration, and invasion under high glucose-induced condition. (a) Under high glucose-induced condition, the expression level of miR22-3p mRNA in AC16 cells was detected by RT-PCR after indicated plasmids were transfected in AC16 cells; (b) AC16 cell viability detection by CCK8 assay under high glucose-induced condition after indicated plasmids were transfected in AC16 cells; (c, d) AC16 cells colony forming ability and proliferation ability detection by colony forming assay and EDU assay of knocking down LncRNA NKILA under high glucose-induced condition, and indicated plasmids were transfected in AC16 cells; (e) AC16 cells apoptosis detection by Annexin V-FITC assay under high glucose-induced condition after indicated plasmids were transfected in AC16 cells; (f) cell apoptosis protein makers expression level detection by western blot assay after transfecting indicated plasmids in AC16 cells and indicated antibodies were added during western blot assay. Data are representative of three independent experiments and were analyzed by the unpaired $t$-test. Error bars denote SD. ${ }^{*} P<0.05 ;{ }^{* *} P<0.01$; ${ }^{* * *} P<0.001$.

3.5. TXNIP Is a Target of miR-22-3p in AC16 Cells. We then asked the molecular mechanisms underlying the miR-22$3 \mathrm{p}$-induced proliferation, migration, and invasion of AC16 cells. Through searching starBase, aligned region was found between sequence of miR-22-3p and TXNIP forward chain (Figure 7(a)). Furthermore, we utilized luciferase activity assay to analyze the interaction between miR-22-3p and TXNIP. miR-22-3p overexpressing (miR-22-3p mimic) significantly inhibited TXNIP luciferase activity compared with control group (NC mimic), which was abolished by TXNIP mutation (Figure $7(\mathrm{a})$ ), suggesting TXNIP is a target of miR-22-3p (Figure 7(b)). The expression level of TXNIP mRNA increased significantly with the increase in the time of high glucose induction (Figure 7(c)), and the increase in TXNIP mRNA expression level was dose-dependent on the glucose concentration (Figure $7(\mathrm{~d})$ ). And compared with control group (NC mimic), miR-22-3p overexpressing (miR-22-3p mimic) significantly inhibited TXNIP mRNA and protein expression level (Figures 7(e) and 7(f)), suggesting TXNIP is a target of miR-22-3p.

3.6. LncRNA NKILA Performed Its Function in AC16 Cells under High Glucose-Induced Condition by Targeting miR-22-3p-TXNIP Signal Axis. The above data demonstrated that TXNIP was a target of miR-22-3p, indicating LncRNA NKILA performed its function in AC16 cells under high glucose-induced condition targeting miR-22-3p-TXNIP signal axis. To prove this hypothesis, we first validated the efficiency of LncRNA NKILA shRNA (Figure 8(a)). Then, we performed CCK8 assay and found that, under high glucose-induced condition, knocking down LncRNA NKILA $(\mathrm{HG}+$ sh-NKILA + NC inhibitor) significantly increased AC16 cells viability compared with control group ( $\mathrm{HG}+\mathrm{sh}-\mathrm{NC}+\mathrm{NC}$ inhibitor) (Figure $8(\mathrm{~b})$ ). And this kind degree increase in cell viability was inhibited when miR-22-3p inhibitor was added $(\mathrm{HG}+\mathrm{sh}-\mathrm{NKILA}+\mathrm{miR}-22-3 \mathrm{p}$ inhibitor $)$, which could be rescued by knocking down TXNIP (HG + sh-NKILA + miR22-3p inhibitor + sh-TXNIP) (Figure $8(b)$ ). To evaluate cell proliferation, we performed EDU assay and found that, under high glucose-induced condition, knocking down LncRNA NKILA (HG + sh-NKILA + NC inhibitor) significantly inhibited AC16 cells proliferation compared with control group (HG + sh-NKILA $+\mathrm{NC}$ inhibitor) (Figure $8(\mathrm{c})$ ). And this kind degree inhibition of cell proliferation was restored when miR-22-3p inhibitor was added $(\mathrm{HG}+\mathrm{sh}-\mathrm{NKILA}+\mathrm{miR}-22-3 \mathrm{p}$ inhibitor $)$, which could be recorded by knocking down TXNIP (HG+sh-NKI$\mathrm{LA}+\mathrm{miR}-22-3 \mathrm{p}$ inhibitor $+\mathrm{sh}-\mathrm{TXNIP})$ (Figure 8(c)). When we performed Annexin V-FITC assay, these results were 

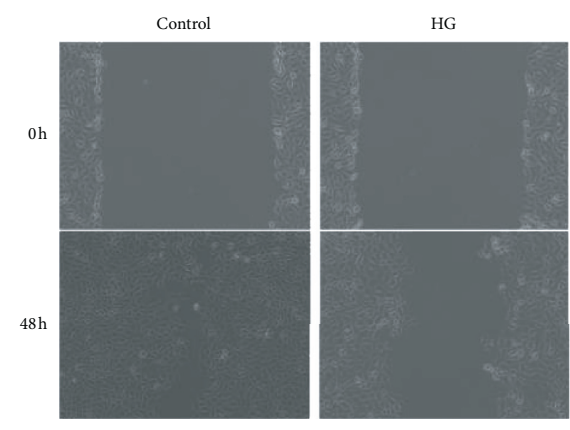

HG

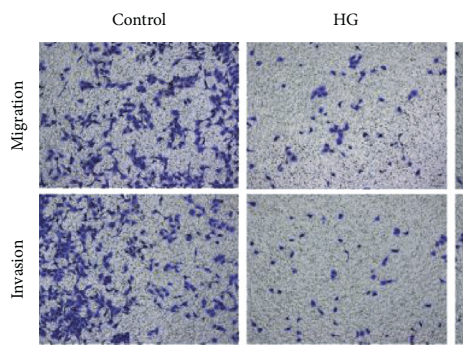

$\mathrm{HG}+\mathrm{NC}$ mimic

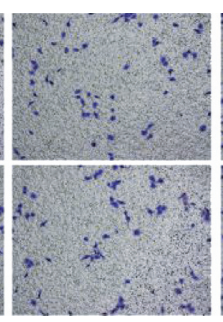

$\mathrm{HG}+\mathrm{NC}$ mimic

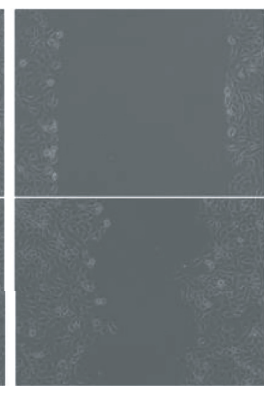

(a)

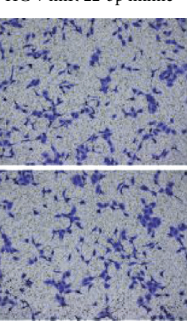

(b)
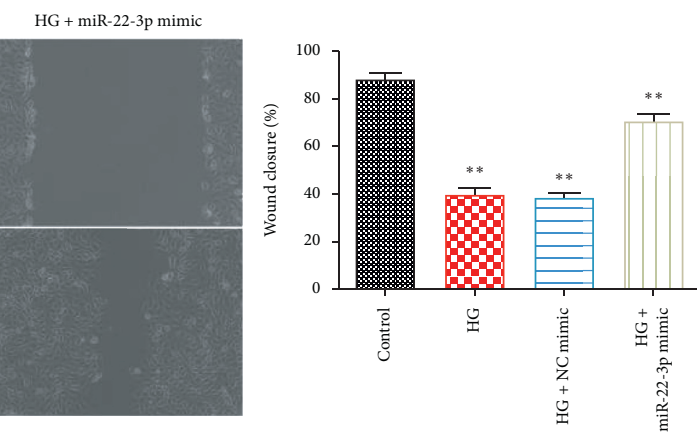

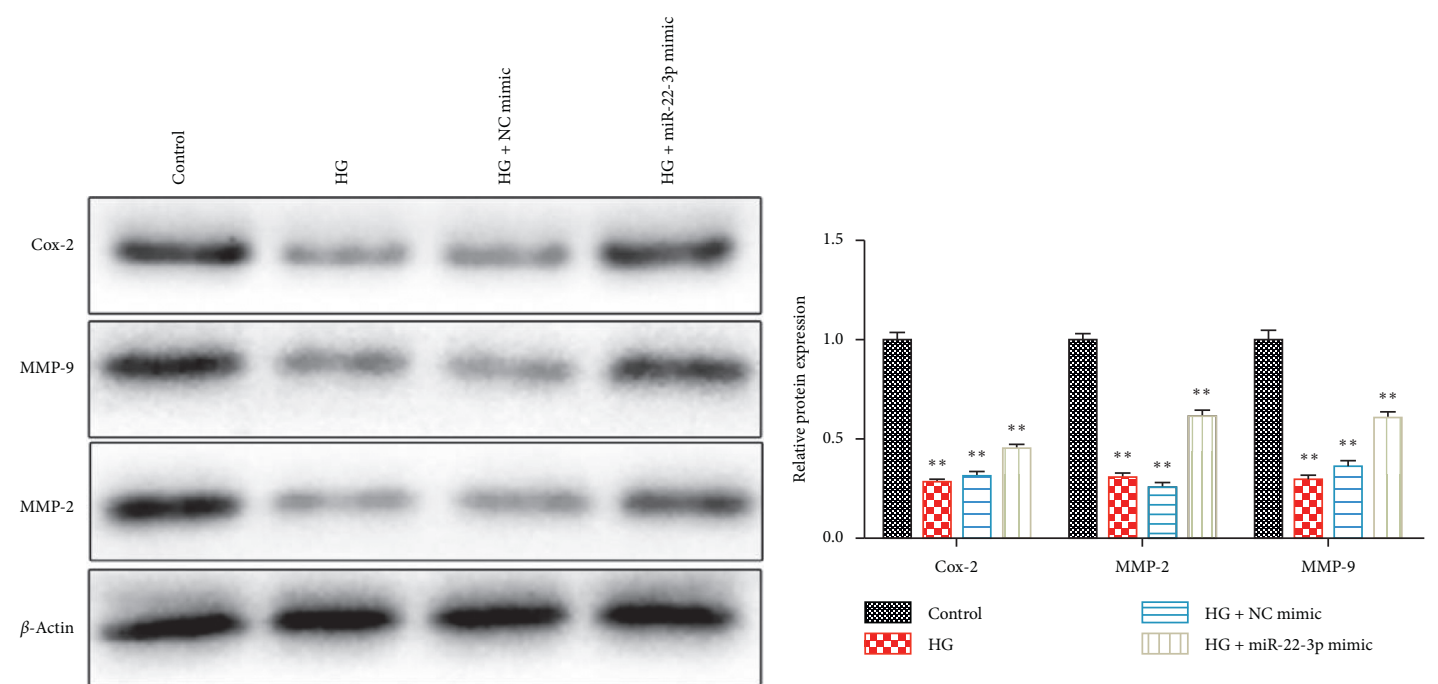

(c)

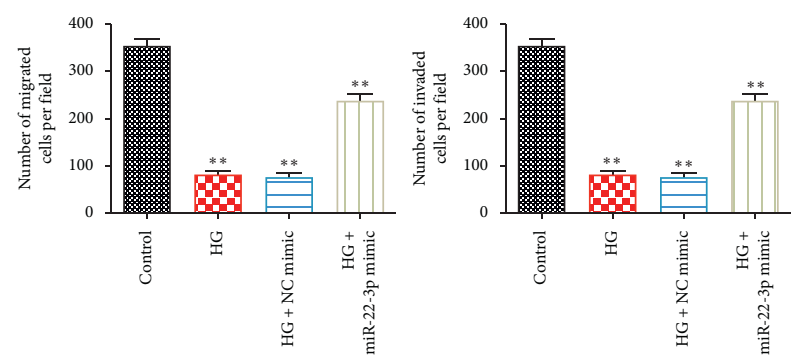

FIGURE 6: MiR22-3p affect on migration and invasion of AC16 cells under high glucose-induced condition. (a, b) AC16 cells migration and invasion detection by the wound healing test chamber assay under high glucose-induced condition after transfecting indicated plasmids in AC16 cells; (c) cell migration and invasion protein makers expression level detection by western blot assay after transfecting indicated plasmids in AC16 cells and indicated antibodies were added during the western blot assay. Data are representative of three independent experiments and were analyzed by the unpaired $t$-test. Error bars denote SD. ${ }^{*} P<0.05 ;{ }^{* *} P<0.01 ;{ }^{* * *} P<0.001$.

observed in AC16 cells apoptosis as well (Figure 8(d)). Next, we performed wound healing test and transwell chamber assay to evaluate influence on migration and invasion. As shown in Figures 8(e) and 8(f), under high glucose-induced condition, knocking down LncRNA NKILA (HG + shNKILA + NC inhibitor) significantly increased AC16 cells migration and invasion compared with control group $(\mathrm{HG}+$ sh-NC $+\mathrm{NC}$ inhibitor) (Figures $8(\mathrm{e})$ and $8(\mathrm{f}))$. And this kind degree increase in cell migration and invasion was inhibited when miR-22-3p inhibitor was added ( $\mathrm{HG}+\mathrm{sh}$ NKILA + miR-22-3p inhibitor), which could be rescued by knocking down TXNIP (HG + sh-NKILA + miR-22-3p inhibitor + sh-TXNIP) (Figures $8(\mathrm{e})$ and $8(\mathrm{f})$ ). Taken together, these data indicated that LncRNA NKILA performed its function in AC16 cells under high glucose-induced condition by targeting miR-22-3p-TXNIP signal axis. 


\begin{tabular}{|c|c|}
\hline TXNIP WT & $5^{\prime}$-uauuggccccucacuGCAGCUg- $3^{\prime}$ \\
\hline Hsa-miR-22-3p & $3^{\prime}$-ugucaagaaguugacCGUCGAa- $5^{\prime}$ \\
\hline TXNIP Mut & $5^{\prime}$-uauuggccccucacuGCAGCUg- $3^{\prime}$ \\
\hline
\end{tabular}

(a)

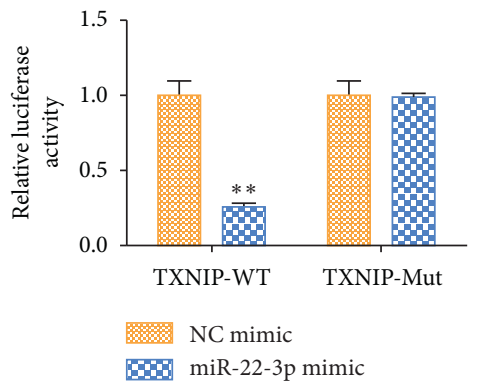

(b)

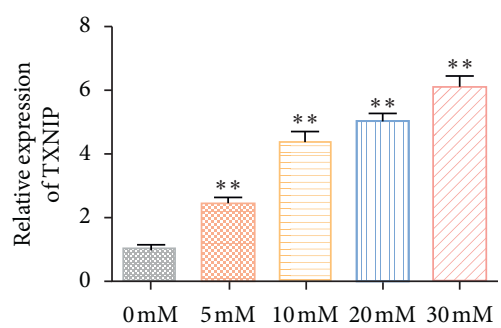

(d)

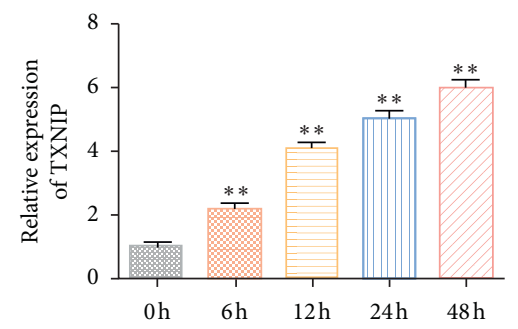

(c)

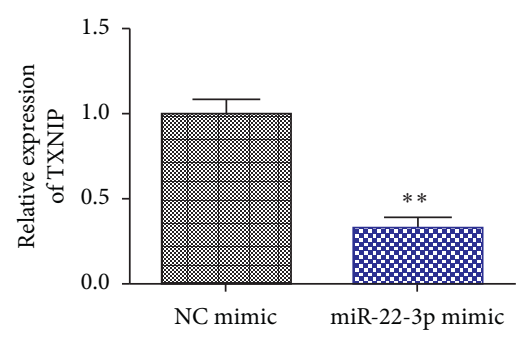

(e)
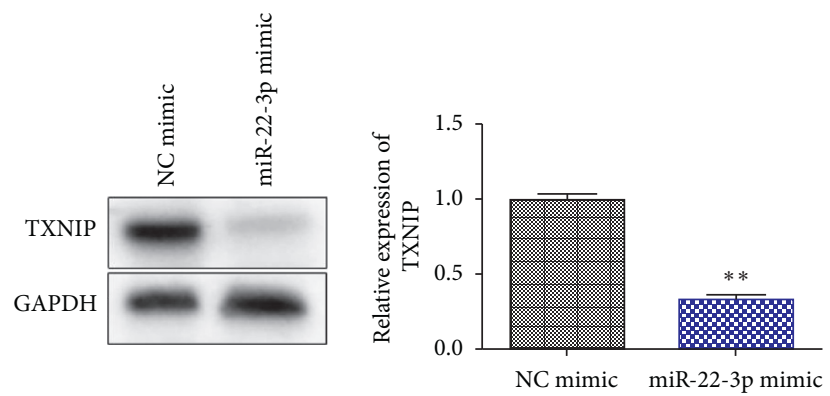

(f)

FIgURE 7: TXNIP is a target of miR-22-3p in AC16 cells. (a) Aligned region between sequence of TXNIP and miR22-3p and sequence of TXNIP mutation(TXNIP Mut); (b) luciferase activity assay to analyze the interaction between TXNIP (wt: wild type or Mut: mutation) and miR22-3p, under high glucose-induced condition; (c) the expression level of TXNIP mRNA detected by RT-PCR after inducing on $30 \mathrm{mM}$ glucose at the indicated time in AC16 cells; (d) the expression level of TXNIP mRNA detected by RT-PCR after inducing with various concentrations of glucose $(0,5,10,20$, and $30 \mathrm{mM})$ for $48 \mathrm{~h}$ in AC16 cells; (e, f) TXNIP mRNA and protein expression level inhibited by miR22-3p mRNA, mRNA, and protein expression level were detected by RT-PCR and western blot assay after transfecting indicated plasmids in AC16 cells under high glucose-induced condition and indicated antibodies were added during western blot assay. Data are representative of three independent experiments and were analyzed by the unpaired $t$-test. Error bars denote SD. ${ }^{*} P<0.05 ;{ }^{* *} P<0.01 ;{ }^{* * *} P<0.001$.

\section{Discussion}

Despite significant advances in the clinical treatment of DCM in recent years, the mortality rate among DCM patients is still unacceptably high, resulting in it being one of the leading causes of death from diabetic complication-related diseases worldwide [24]. The difficulties in the treatment of patients with DCM stem from our limited understanding of its pathogenesis at the molecular level, which has been an area of intensive research in recent years. Elucidating the molecular mechanism controlling pathogenesis of DCM is of great importance to DCM.

Several evidences have been provided in the past on the regulatory role of $\operatorname{lncRNAs}$ in heart development and diseases [10]. At present, LncRNA NKILA has been extensively studied for its anticancer effect in several malignancies. To the best of our knowledge, the involvement of lncRNA NKILA in diabetic complications remains poorly understood. Apoptosis of 


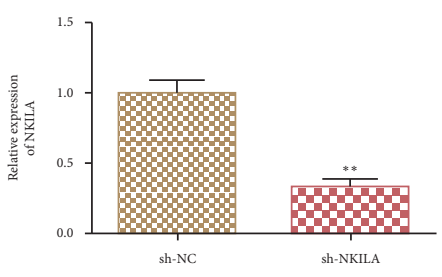

(a)

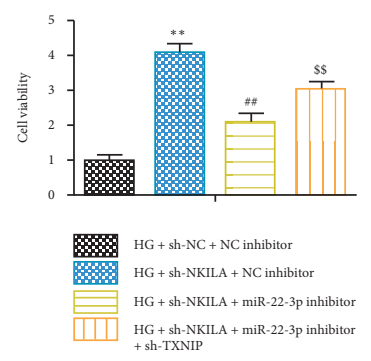

(b)
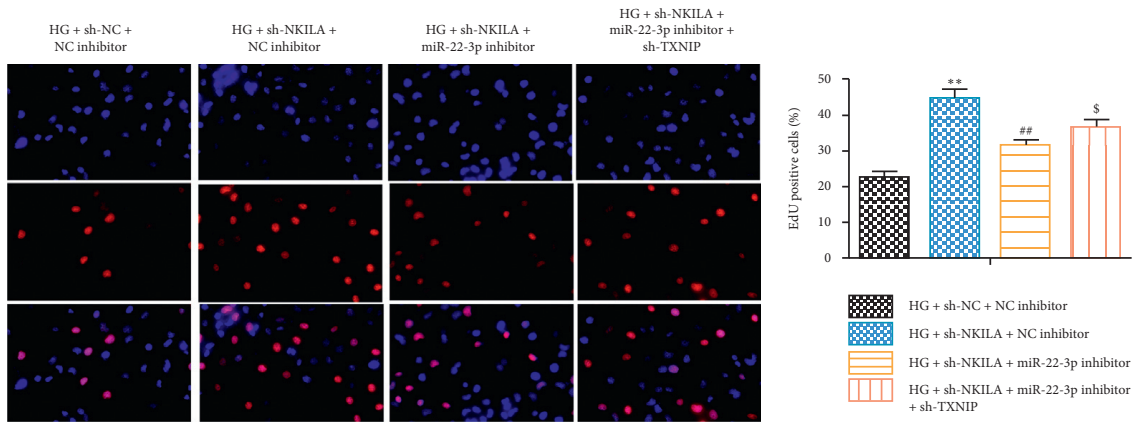

(c)
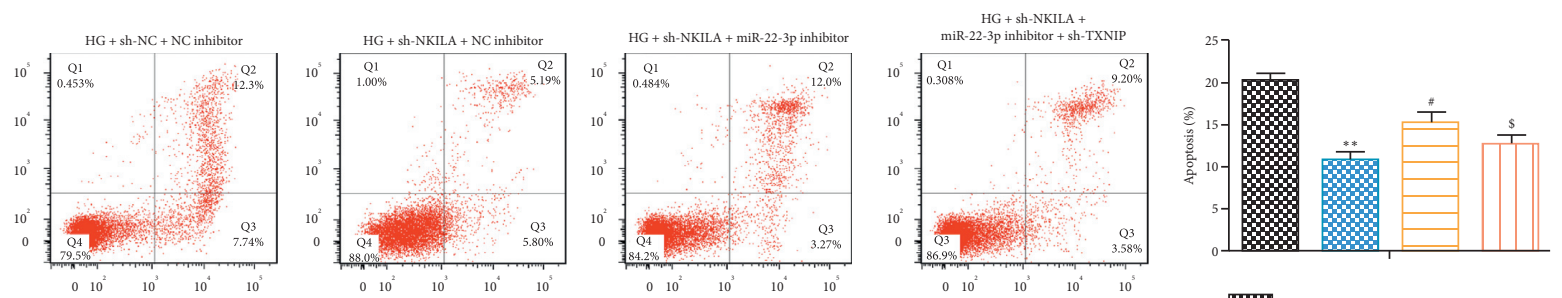

\% $\mathrm{HG}+$ sh-NC $+\mathrm{NC}$ inhibitor

17. $\mathrm{HG}+$ sh-NKILA + NC inhibitoe HG + sh-NKILA + miR-22-3p inhibitor

III HG + +h-NKILA + miR-22-3p inhibitor

(d)
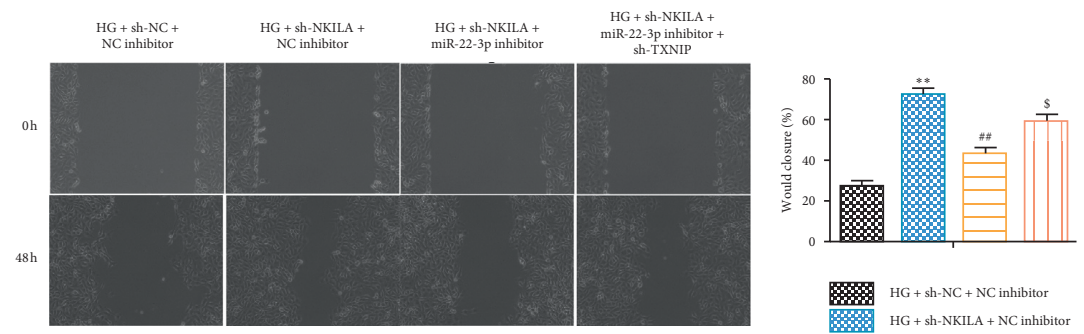

\% $\mathrm{HG}+$ sh-NC + NC inhibito

HG + sh-NKILA + NC in

HG + sh-NKILA + miR-22-3p inhibito

$\underset{\substack{\text { HG + sh-NKILA + miR-22-3p inhibitor } \\+ \text { sh-TXNIP }}}{\substack{11 \\ \text { s. }}}$

(e)

FIgure 8: Continued. 


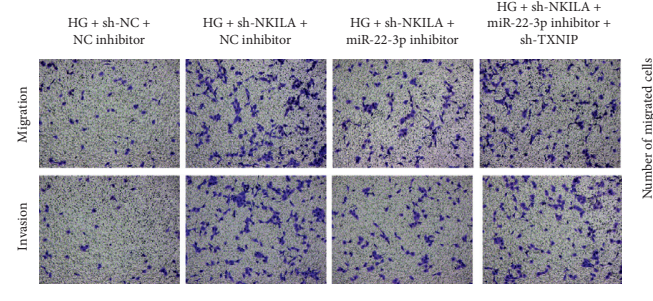

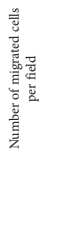

(f)

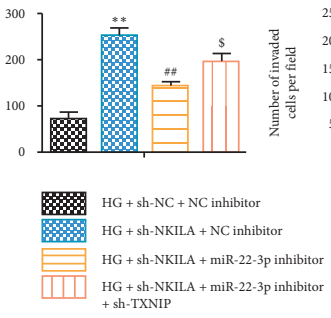

HG + sh-NKILA + miR-22-3p inhibitor

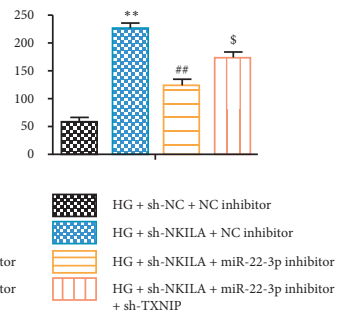

FIgURE 8: LncRNA NKILA performed its function in AC16 cells under high glucose-induced condition by targeting mir-22-3p-TXNIP signal axis. (a) LncRNA NKILA mRNA expression level was detected by RT-PCR after transfecting indicated plasmids in AC16 cells under high glucose (b) and (c) AC16 cell viability and proliferation ability detection by CCK8 assay and EDU assay under high glucose=induced condition after indicated plasmids were transfected in AC16 cells; (d) AC16 cells apoptosis detection by Annexin V-FITC assay under high glucose-induced condition after indicated plasmids were transfected in AC16 cells; (e, f) AC16 cells migration and invasion detection by the wound healing test and transwall chamber assay under high glucose-induced condition after transfecting indicated plasmids in AC16 cells. Data are representative of three independent experiments and were analyzed by the unpaired $t$-test. Error bars denote SD. ${ }^{*} P<0.05 ;{ }^{* *} P<0.01 ;{ }^{* * *} P<0.001$.

cardiomyocytes in high glucose environment is involved in the pathogenesis of DCM [25], and inhibition of this pathophysiological process is considered as a promising therapeutic target of DCM [26]. In our study, we detected lncRNA NKILA expression relationship with high glucose-induced condition in DCM cells. The expression of lncRNA NKILA level in DCM cells was significantly upregulated under high glucose-induced condition. And lncRNA NKILA knockdown accelerated and inhibited apoptosis in cardiomyocytes under high glucose-induced condition. Furthermore, our findings demonstrated that lncRNA NKILA promoted DCM cells apoptosis and inhibited DCM cells proliferation, migration, and invasion in a miR-223 -dependent way. We also demonstrated that lncRNA NKILA could directly bind to miR-22-3p, which endowed miR22-3p with the ability to reverse the influence of LncRNA NKILA on DCM cells proliferation, migration, and invasion. Luciferase activity assay demonstrated that TXNIP was a target of miR-22$3 p$ in DCM cells; therefore, all the affect of TXNIP on DCM cells proliferation, migration, and invasion could be abolished by miR22-3p.

In conclusion, the present study demonstrated a novel molecular mechanism of LncRNA NKILA of promoting cardiomyocytes apoptosis: LncRNA NKILA performed its function in DCM cells under high glucose-induced condition by targeting miR-22-3p-TXNIP signal axis, which indicated that LncRNA NKILA may play a crucial role in diabetic cardiomyopathy. Taken together, our data suggested that LncRNA NKILA may be a promising therapeutic target for treating DCM.

\section{Data Availability}

All data used to support the findings of this study are included within the manuscript.

\section{Conflicts of Interest}

The authors declare that there are no conflicts of interest.

\section{Acknowledgments}

This work was supported by the Shanghai Pudong New Area Summit (Emergency Medicine and Critical Care) Construction Project (Grant no. PWYgf2018-05).

\section{References}

[1] J. M. Forbes and M. E. Cooper, "Mechanisms of diabetic complications," Physiological Reviews, vol. 93, no. 1, pp. 137-188, 2013.

[2] W. Bao, D.-S. Huang, and Y.-H. Chen, "MSIT: malonylation sites identification tree," Current Bioinformatics, vol. 15, no. 1, pp. 59-67, 2020.

[3] J. C. Kovacic, S. Dimmeler, R. P. Harvey et al., "Endothelial to mesenchymal transition in cardiovascular disease," Journal of the American College of Cardiology, vol. 73, no. 2, pp. 190-209, 2019.

[4] R. H. Ritchie, E. J. Zerenturk, D. Prakoso, and A. C. Calkin, "Lipid metabolism and its implications for type 1 diabetesassociated cardiomyopathy," Journal of Molecular Endocrinology, vol. 58, no. 4, pp. R225-R240, 2017.

[5] S. Sharma, J. V. Adrogue, L. Golfman et al., "Intramyocardial lipid accumulation in the failing human heart resembles the lipotoxic rat heart," The FASEB Journal, vol. 18, no. 14, pp. 1692-1700, 2004.

[6] M. U. Qazi and S. Malik, "Diabetes and cardiovascular disease: insights from the framingham heart study," Global Heart, vol. 8, no. 1, pp. 43-48, 2013.

[7] Y. Wang, Y. Wang, F. Li et al., "Spermine protects cardiomyocytes from high glucose-induced energy disturbance by targeting the CaSR-gp78-ubiquitin proteasome system," Cardiovascular Drugs and Therapy, vol. 35, 2020.

[8] W. Bao, B. Yang, D. Li, Z. Li, Y. Zhou, and R. Bao, "CMSENN: computational modification sites with ensemble neural network," Chemometrics and Intelligent Laboratory Systems, vol. 185, pp. 65-72, 2019.

[9] W. Bao, B. Yang, D.-S. Huang et al., "IMKPse: identification of protein malonylation sites by the key features into general PseAAC," IEEE Access, vol. 7, pp. 54073-54083, 2019. 
[10] W. Bao, C.-A. Yuan, Y. Zhang et al., "Mutli-features predction of protein translational modification sites," IEEE/ACM Transactions on Computational Biology and Bioinformatics, vol. 15, no. 5, pp. 1453-1460, 2018.

[11] M. Zhang, H. Gu, J. Chen, and X. Zhou, "Involvement of long noncoding RNA MALAT1 in the pathogenesis of diabetic cardiomyopathy," International Journal of Cardiology, vol. 202, pp. 753-755, 2016.

[12] L. Bird, "IncRNA NKILA: a killer regulator," Nature Reviews Immunology, vol. 18, no. 11, pp. 666-667, 2018.

[13] R. Chen, Q. Cheng, K. G. Owusu-Ansah et al., "NKILA, a prognostic indicator, inhibits tumor metastasis by suppressing NF- $\kappa \mathrm{B} /$ Slug mediated epithelial-mesenchymal transition in hepatocellular carcinoma," International Journal of Biological Sciences, vol. 16, no. 3, pp. 495-503, 2020.

[14] L.-H. Luo, L. Rao, L.-F. Luo, K. Chen, R.-Z. Ran, and X.-L. Liu, "Long non-coding RNA NKILA inhibited angiogenesis of breast cancer through NF- $\kappa$ B/IL-6 signaling pathway," Microvascular Research, vol. 129, p. 103968, 2020.

[15] W. Bao, D. Wang, and Y. Chen, "Classification of protein structure classes on flexible neutral tree," IEEE/ACM Transactions on Computational Biology and Bioinformatics, vol. 14, no. 5, pp. 1122-1133, 2017.

[16] J. C. Chatham, M. E. Young, and J. Zhang, "Role of O-linked $\mathrm{N}$-acetylglucosamine (O-GlcNAc) modification of proteins in diabetic cardiovascular complications," Current Opinion in Pharmacology, vol. 57, pp. 1-12, 2020.

[17] J. Russell, E. F. Du Toit, J. N. Peart, H. H. Patel, and J. P. Headrick, "Myocyte membrane and microdomain modifications in diabetes: determinants of ischemic tolerance and cardioprotection," Cardiovascular Diabetology, vol. 16, no. 1, p. 155, 2017.

[18] W. Bao, D. S. Huang, C. A. YuanHuang, D. S. Huang et al., Pupylation sites prediction with ensemble classification model," International Journal of Data Mining and Bioinformatics, vol. 18, no. 2, pp. 91-104, 2017.

[19] A. Villard, L. Marchand, C. Thivolet, and S. Rome, "Diagnostic value of cell-free circulating MicroRNAs for obesity and type 2 diabetes: a meta-analysis," Journal of Molecular Biomarkers \& Diagnosis, vol. 6, no. 6, 2015.

[20] J. C. Chambers, M. Loh, B. Lehne et al., "Epigenome-wide association of DNA methylation markers in peripheral blood from Indian Asians and Europeans with incident type 2 diabetes: a nested case-control study," The Lancet Diabetes \& Endocrinology, vol. 3, no. 7, pp. 526-534, 2015.

[21] C.-R. Chong, W. P. A. Chan, T. H. Nguyen et al., "Thioredoxin-interacting protein: pathophysiology and emerging pharmacotherapeutics in cardiovascular disease and diabetes," Cardiovascular Drugs and Therapy, vol. 28, no. 4, pp. 347-360, 2014.

[22] T. Hara, B. Abdulaziz Umaru, K. Sharifi, T. Yoshikawa, Y. Owada, and Y. Kagawa, "Fatty acid binding protein 7 is involved in the proliferation of reactive astrocytes, but not in cell migration and polarity," Acta histochemica et cytochemica, vol. 53 , no. 4 , pp. 73-81, 2020.

[23] T. Clément, V. Salone, and M. Rederstorff, "Dual luciferase gene reporter assays to study miRNA function," Methods in Molecular Biology, vol. 1296, pp. 187-198, 2015.

[24] G. Jia, A. Whaley-Connell, and J. R. Sowers, "Diabetic cardiomyopathy: a hyperglycaemia- and insulin-resistance-induced heart disease," Diabetologia, vol. 61, no. 1, pp. 21-28, 2018.

[25] W. Wu, X. Liu, and L. Han, "Apoptosis of cardiomyocytes in diabetic cardiomyopathy involves overexpression of glycogen synthase kinase-3 $\beta$," Bioscience Reports, vol. 39, no. 1, 2019.
[26] J. Du, S. T. Yang, J. Liu, K. X. Zhang, and J. Y. Leng, "GAS5Silence of LncRNA protects cardiomyocytes H9c2 against hypoxic injury via sponging," Molecules and Cells, vol. 42, no. 5, pp. 397-405, 2019. 